\title{
The Book of Life goes online
}

\author{
PETTER HOLM ${ }^{1}$
}

\section{A walk in the park}

It's a beautiful day for the great outdoors. You have decided to take a break from city life. Sunny fall weather; it's drying up from the rain last week. Perfect for picking mushrooms. You bring a basket, small brush, sharp knife. And the biopod, the latest generation life barcoder, 'Tricorder' edition. ${ }^{2}$ After a short walk to your own secret mushroom place, you spot a patch of nice-looking specimens. Caps are 5-10 cm across, with slightly depressed centres. Slightly sticky. Colour brownish to dark brickred. Gills close together. It could be the delicious 'Flirt' (Russula vesca). Or is it the poisonous 'Sickener' (Russula emetica)? You quickly scan it with the barcoder. There is a barely-audible hum as the device goes online. A few seconds later, the display shows Russula vesca. Great! Scrolling down the tiny screen, you're informed that its mild flavour goes well with lamb stew. Serve with a light red Italian. You fill the basket, and head to the supermarket for the rest of the ingredients you need to prepare a fine meal.

\section{Linnaeus in the sky}

This sort of scene becomes possible to envisage from Costa and Carvalho's synopsis on the Barcode of life initiative. ${ }^{3}$ At first glance, it has a Star Trek feel to it: a landing party is beamed from the safety of the starship onto some planet "where no one has gone before', equipped with tricorders serving as lifelines and generalised information gadgets. Whereas a real-life version of this scenario might have been dismissed as pure fiction a few years ago, the rapid rise of GSP and mobile "phones have made it more realistic. The Barcode of Life Initiative extends just slightly what is now a familiar scene. Instead of dispensing with a map, compass and navigation skills, as the GPS did, the life barcoder promises easy access to the identity of the wildlife along your track. When one is equipped with such a hand-held device, it is as if the mushroom comes fixed with a label. Instead of the cumbersome task of teaching yourself how to be a taxonomist, or bringing one along from the local museum, you simply consult the virtual Linneaus in the sky.

From Costa and Carvalho's fine review, we already know how the Barcode of Life pulls off this feat. The barcoder analyses DNA from a tissue sample taken from the target specimen and links it to a barcode. With this barcode, the identity of your specimen is fixed as a specific location in a DNA-based species classification system, which also provides easy access to other relevant information, be it, in the mushroom case, the appropriate antidote or the wine that best brings out its flavour. 


\section{Barcoding and the Encyclopedia of Life}

A virtual Linnaeus would be a wonderful thing. Such a system, fully operational, comes with a number of advantages. It can identify species from tissue fragments and regardless of life-history stage. Ambiguity is reduced and identification of look-alikes becomes straightforward. The identification of known species can now be safely left to amateurs, allowing the experts to focus on unknown species.

While Costa and Carvalho are excellent guides to the advantages of a fully functional barcode-of-life system, they are less explicit when it comes to the investment required before the system can go online. How much and what kind of work does it take to make a virtual Linnaeus? The key here is the classification system by which the DNA sequence from a specific genome region is linked to some (hierarchically ordered) list of named species. For a lay person to use a barcoder to identify species, the databases by which these links can be made must already be in place. There must be an 'Encyclopedia of Life', with information linked to barcodes. If a specimen's barcode is not registered, the amateur will remain uninformed.

This problem is comparable with one commonly encountered in supermarket checkout queues, when an item - usually from the fruit and vegetable section - lacks a barcode. When the cashier is confronted with a species exotic to him - is this a cantaloupe or a galia? - he must become an old-fashioned taxonomist, consulting, first, the super-market version of a field-guide. If this is unsuccessful, he must call up a real expert from the back of the shop somewhere to identify it. Only then can you pay for your merchandise and take it away with you.

A sizable supermarket contains around 50,000 items. Here, classification is reasonably easy: not only are there relatively few species, but all have been classified and priced prior to sale. The problem is not one of knowing the identity of your species, but making that information available at the checkout. Nature, by comparison, is a far grander kind of supermarket, storing many millions of items. Here, the inventory is not pre-established, but must be built from scratch. While the barcoder allows the lay person easy access to the labelled checked entries, there remains a problem with the un-labelled species. Since the barcode is encoded in the specimen itself, you will of course always get a reading. But if the species in question has not already been named and entered into the Encyclopedia of Life, your query will remain unanswered.

\section{Setting up an Open Writing Workshop}

A complete Encyclopedia of Life is not the only advantage of the Barcode of Life Initiative. Another major attraction is the ease by which entries can be added. To use Costa and Carvalho's vocabulary, barcoding will speed up species delineation as well as species identification. 
Assume the Initiative produces the first draft of a DNA barcode 'Encyclopedia of Life' by adding the relevant DNA key to the list of all conventionally named and classified species. In the process, improvements such as the definition of the true identity of similar species will already have been made. Nevertheless, the basic problem is much the same as before, namely that most species remain unclassified. How could the DNA barcoding technology help fill in the blanks?

The first point emphasised by Costa and Carvalho concerns the economy of expertise. Since barcoding makes identification of labelled species easy, the professional taxonomists can concentrate their work on the uncharted territories. However, the taxonomist community should perhaps not rely on this, since the number of taxonomists in society is not a constant. Can we assume that resources freed up by an efficient species identification technology will be allocated to the task of species delineation? Given the tight budgets of the organizations employing taxonomists, and the constant struggle among worthy causes, the answer to this is unclear.

Another, perhaps more interesting point, concerns how a barcode classification system allows for the process of species delineation to be organised differently. Imagine that a cluster of unknown barcode readings has been reported by reliable sources, which leads to the formulation of a hypothesis of a new species. While the rejection or confirmation of such hypotheses will still require expert opinion, the barcoding technology invites broad participation in the collection of the information required to test it. Working from the fixed point of DNA-based identification, a protocol on data collection can be set up and distributed. In this way, authorship of the Encyclopedia of Life changes. Instead of the expert taxonomist working alone in the dusty dungeons of the museum, the Encyclopedia becomes a collaborative effort involving many different people.

A related point here concerns the status of the conventional taxonomist as expert. Initially, as underlined by Costa and Carvalho, the barcode classification of a given species is a hypothesis to be checked against the conventional classification. The conventional taxonomist's expertise with the tools of the trade mean that he remains first author and gate keeper for DNA-based classification. If and when the technology proves itself, however, this is turned on its head. The conventional classification changes status, and becomes the hypothesis that must be checked against the barcode classification. The real experts, set to judge between true and false Linnean classification, are those who master DNA-based technologies. While this may look problematic from the point of view of today's taxonomy profession, such is the normal destructiveness of progress. Indeed, the re-organisation of the taxonomy profession is an important feature of barcode technology. With the DNA barcode as classification key, folk taxonomy becomes, just as the Linnean classification did before, a new and interesting source of hypotheses for species identity.

\section{A few dark possibilities}

Will the world become a happier and more just place with the success of the Barcode of Life initiative? As always, new technologies produce both winners and losers when 
they are let loose on the real world. A major question here is how barcoding might affect the balance of power in an already unfair world. Who stands to gain: the resource-rich of the North or the impoverished of the South?

Wouldn't it have been nice if the DNA barcoding, on top of everything else, also helped the poor and powerless? But this is not the case. The Encyclopedia of Life - in both its conventional and DNA-barcode versions - is more complete for the North than the South. The broad, democratic access to species identification by way of barcoding technology will therefore be of most relevance in the North. In the South, where most unlabelled species are to be found and the lack of resources to fill the blanks are most obvious, its usefulness is less clear. It could be argued, of course, that the efficiency of DNA-based delineation will give developing countries a chance to make inventories of their natural riches. Another, darker possibility is that such inventories will be of most interest to capitalist firms on bioprospecting excursions. While DNA barcoding may allow indigenous people to be co-authors of the Encyclopedia of Life, it may be the modern pharmaceutical giants that stand to reap the financial rewards.

\section{Do you want to live in a supermarket?}

The rich stand to gain while the poor lose out. In the face of this, the have-nots should organise and fight as best they can. Not to ban the technology, of course, but to reformat it in a way that can serve their interests. While we wait for this upcoming struggle, we can take time to consider whether a barcoded world is desirable. A romantic might put it thus: 'Do you really want to live in a supermarket - a world where every species comes pre-labelled for reading with a handheld device?' To the romantic, the answer is 'no', of course. To him, barcoding is but an extension of the iron cage of rationality, a place where the disenchantment of the world has reached an extreme, and all wilderness has been emptied of mystery and turned into yet another supermarket.

While I acknowledge this fear, however, I do not share it. The wilderness of the world simply cannot be contained in a classification scheme. Just like a map, classification does not really reduce the complexity of the world, but allows you to travel more effectively within it. The barcoder offers a fine meal of 'Flirt' mushrooms instead of violent vomiting induced by the 'Sickener'. Just like Star Trek's tricorder, the barcoder will not prevent the adventure, but serve as a valuable companion for your travel to places where 'no one has gone before.'

\footnotetext{
${ }^{1}$ Norwegian College of Fishery Science, University of Tromsø Petter.Holm@nfh.uit.no

${ }^{2}$ A tricorder is a handheld device used for scanning an area, interpreting and displaying data from scans to the user, and recording information. A tricorder is a prop in the Star Trek Universe. See www.startrek.com

${ }^{3}$ F.O. Costa and G.R. Carvalho. The Barcode of Life Initiative: synopsis and prospective societal impacts of DNA barcoding of fish. Genomics, Society and Policy 2007; 3 (2): 29-40.
} 